G439(P) TALE OF ALLERGY TESTING: IMPLEMENTING RCPCH ALLERGY CARE PATHWAYS IN A DISTRICT GENERAL HOSPITAL

S Bilal, E White, L Bowers, A Shenoy. Paediatrics, Bradford Royal Infirmary, Bradford, UK

10.1136/archdischild-2015-308599.393

Introduction $\mathrm{RCPCH}$ allergy care pathways recommend use of either RAST or skin prick testing for diagnosis only when there is clinical suspicion of allergy. Pathways further recommend use of measurements of skin prick and specific IgE test results to optimise the timing of food challenges. This audit aimed to compare our practice against national standards.

Method A retrospective analysis of all notes for food challenges between May 2013-May 2014 was undertaken. Total of 29 food challenges were identified.

Results More than half of our patients were males. Majority of the patients (69\%) had RAST test done at time of diagnosis. 7\% of patients had both skin prick and RAST done. However, 21\% of allergy patients did not have any test done at time of diagnosis and diagnosis was made on history only.

In regards to food challenge, more then half of patients were aged 8 years or above at time of challenge. Only $66 \%$ of patients passed food challenge. $79 \%$ of patients have either RAST or Skin prick test done prior to challenge. $21 \%$ of our patients had no form of testing prior to challenge. Of all patients who passed challenge $53 \%$ of patients have both tests done in contrast to all patients who failed challenge only $30 \%$ have both tests done. All patients who had both tests negative passed the food challenge. All the patients who failed the challenge had a positive RAST test prior to challenge.

Recommendations An allergy clinic proforma was introduced as an aide-memoir to facilitate appropriate testing at diagnosis and prior to challenge.

Failed food challenges bring disappointment for child, parents and unnecessary burden on NHS budget. Choosing the right patient is the key to prevent failed challenges, hence, referral process for challenges was re evaluated and now clinicians will make sure that both tests are done prior to food challenges.

We aim to re audit in six months.

\section{G440(P) TO AUDIT THE MANAGEMENT OF CLOSTRIDIUM DIFFICILE (C. DIFFICILE) INFECTION IN CHILDREN}

R Atterby, S Bandi. Childrens Hospital, Leicester Royal Infirmary, Leicester, UK

\subsection{6/archdischild-2015-308599.394}

Methods C. difficile toxin positive cases were identified using the laboratory data between 01.01 .12 and 31.12 .13 . Notes were reviewed and data collected using a proforma against the hospital guideline. The guideline also states that all positive cases should have a MDT review.

Results 26 C. difficile toxin positive cases were identified from 17 patients. Mean age was 6yrs 5 months, with $92 \%>24$ months at time of diagnosis. 21 were included in the final analysis as medical notes weren't available for 5 patients.

Risk factors

$47 \%$ had documentation of recent/current course of antibiotics.

$32 \%$ of cases identified had previous c difficile toxin positive samples.

7 positive results were from oncology patients

Management
- 58\% (12/21) were treated with antibiotics for what were considered clinically significant infections

- There was 100\% compliance with the guidelines regarding antibiotic choice (metronidazole first line, vancomycin second line).

- $41 \%(5 / 12)$ cases were treated for the recommended 10 day duration. $16 \%(2 / 12)$ patients received antibiotics for 14 days. $33 \%(4 / 12)$ had variable treatment duration ranging between 1-7 days.

- There was no documented review of medication for those on either PPI or laxatives and of those on antibiotics only $43 \%$ had documentation of review with the result of the c. difficile test.

MDT outcome vs Treated cases

- 66\% (8/12) 'not confirmed' (3 had complete course in spite of MDT review; 1 discontinued after review; 1 patient died due to underlying co-morbidities; 3 did not receive antibiotics)

- 33\% (4/12) 'confirmed' (all treated with full course of antibiotics)

Repeat stool samples were requested in $26 \%$ within 4 weeks of the positive result.

Conclusion C. Difficile testing presents a challenge for paediatricians. The guidelines are mainly adult focussed. More than half of our positive patients didn't need treatment. The audit highlights the importance of appropriate sampling and the vital role of multidisciplinary team (paediatricians, microbiologists and infection prevention team) in managing these patients.

Recommendation A proforma has been designed to help improve the management of these patients.

\section{G441(P) PLEASE SIR, WHICH IS THE BEST SCHOOL ALLERGY CARE PLAN?}

JC Mack, MA Lewis, D Tuthill, E Spear. General Paediatrics, Cardiff and Vale University Health Board, Cardiff, UK

\subsection{6/archdischild-2015-308599.395}

Introduction Multidisciplinary allergy school care plans are important to manage children with allergies safely in school. They focus on allergen avoidance and treatment of reactions. The Cardiff children's allergy service have developed and used their own plan for 20 years. A new national allergy care plan has been published by BSACI (British Society of Allergy and Clinical Immunology).

Aim To compare Cardiff plan with the BSACI anaphylaxis plan. Methods Based on the NICE guideline for Anaphylaxis (2011) recommendation on information provision, a questionnaire was devised auditing 9 different components of the care plans for clarity and effectiveness. We surveyed teachers, parents, and school nurses in person; respondents completed the questionnaire comparing the two plans. Parents and teachers data (nonhealthcare professionals) was compared to school nurses. Data was analysed with Chi Squared analysis with $\mathrm{P}<0.05$ taken as significant.

Results There were 111 participants (31 teachers, 40 parents and 40 school nurses). The table shows respondents' preferences. For brevity "not answered" and "no preference" responses are not shown.

Conclusion Overall parents and teachers preferred the BSACI plan's succinct format and Epipen usage explanation. In contrast school nurses preferred the Cardiff plan for the same reason! The Cardiff action plan was preferred by most respondents for its clearer documentation of identity, contact details, and 